\title{
MicroRNA-142-3p inhibits cell proliferation and chemoresistance in ovarian cancer via targeting sirtuin 1
}

\author{
JIANLIAN GAO ${ }^{1}$, NAN WU $^{1}$, XIAOHONG LIU ${ }^{1}$, YUECHONG XIA $^{2}$, \\ YING CHEN $^{1}$, SHAORU LI ${ }^{3}$ and ZHIJIAN DENG ${ }^{1}$ \\ ${ }^{1}$ Department of Pharmaceutics; ${ }^{2}$ Intensive Care Unit; ${ }^{3}$ Department of Obstetrics and Gynecology, The \\ First Affiliated Hospital of Xinxiang Medical University, Weihui, Henan 453100, P.R. China
}

Received August 24, 2017; Accepted February 8, 2018

DOI: $10.3892 / \mathrm{etm} .2018 .6107$

\begin{abstract}
MicroRNAs (miRs) serve promoting or suppressive roles in various human cancer types, including ovarian cancer; however, the role of miR-142-3p in ovarian cancer growth and chemoresistance has not previously been studied. In the present study, reverse transcription-quantitative polymerase chain reaction and western blotting were used to examine $\mathrm{miR}$ and protein expression levels. An MTT assay was used to examine cell proliferation. A luciferase reporter gene assay was used to clarify the target gene of miR-142-3p. The present study reported that miR-142-3p expression levels were significantly lower in ovarian cancer tissues and cell lines, when compared with those in adjacent tissues and the normal human ovarian epithelial cell line IOSE386, respectively. The reduced expression of miR-142-3p was significantly associated with poor cell differentiation. Ectopic expression of miR-142-3p significantly inhibited the proliferation of ovarian cancer cells and increased the sensitivity of SKOV3/DDP cells to cisplatin. Sirtuin 1 (SIRT1) was identified as a target gene of miR-142-3p; SIRT1 expression was negatively regulated by miR-142-3p in ovarian cancer cells. Further investigation demonstrated that SIRT1 reversed the suppressive effects of miR-142-3p on the proliferation and chemoresistance of ovarian cancer cells. In addition, SIRT1 was significantly upregulated in ovarian cancer. A negative correlation between the expression of SIRT1 and miR-142-3p in ovarian cancer tissues was also observed. In summary, the present study indicated that miR-142-3p inhibits the proliferation and chemoresistance of ovarian cancer cells by targeting SIRT1. This suggests that miR-142-3p may be a promising therapeutic candidate for the treatment of ovarian cancer.
\end{abstract}

Correspondence to: Professor Zhijian Deng, Department of Pharmaceutics, The First Affiliated Hospital of Xinxiang Medical University, 88 Jiankang Road, Weihui, Henan 453100, P.R. China E-mail: dengzhijian2016@sina.com

Key words: ovarian cancer, microRNA-142-3p, proliferation, chemoresistance, sirtuin 1

\section{Introduction}

Ovarian cancer is one of the most common human malignancies in females, resulting in a high rate of mortality (1). Extensive efforts have been made to improve treatment; however, the survival rate for patients with chemoresistant ovarian cancer remains low (2-4). It has been reported that certain oncogenes and tumor suppressors are dysregulated and serve critical functions during the development and progression of ovarian cancer, and some of these have been suggested to be promising therapeutic targets or candidates $(5,6)$. Thus, investigations into the functions of these genes appear to be beneficial for developing novel strategies for the treatment of ovarian cancer.

MicroRNAs (miRs), a class of non-coding RNAs 18-25 nucleotides in length, inhibit the expression of their target genes by binding to the 3'-untranslated regions (3'UTRs) of their target mRNAs, inducing mRNA degradation or protein translation inhibition $(7,8)$. It has been well documented that miRs are involved in the regulation of numerous cellular processes, including cell survival, differentiation, proliferation and motility (8-11). Additionally, numerous miRs have been reported to be involved in the malignant phenotypes of cancer cells, including cancer cell proliferation, migration, invasion and chemoresistance, by negatively regulating the expression of oncogenes or tumor suppressors (12-15). miR-142-3p has been indicated to act as a tumor suppressor in several common cancer types (16-18). For instance, the downregulation of miR-142-3p may contribute to thyroid follicular tumorigenesis by targeting ASH1 like histone lysine methyltransferase and histone-lysine-N-methyltransferase $2 \mathrm{~A}$ (16). However, the underlying mechanism of miR-142-3p in ovarian cancer is yet to be investigated.

Sirtuin 1 (SIRT1) is a member of the sirtuin family of proteins and functions as an intracellular regulatory protein with mono-ADP-ribosyltransferase and deacetylase activity (19). Recently, Shuang et al (20) reported that SIRT1 overexpression contributed to chemoresistance and poor prognosis in serous epithelial ovarian cancer. In addition, Mvunta et al (21) revealed that SIRT1 also promoted ovarian cancer cell invasion. Therefore, SIRT1 functions as an oncogene in ovarian cancer; however, the regulatory mechanism of SIRT1 expression is largely unknown. 
The present study aimed to investigate the expression of miR-142-3p in ovarian cancer, as well as the molecular mechanism of miR-142-3p underlying the proliferation and chemoresistance of ovarian cancer cells.

\section{Materials and methods}

Tissue collection. The present study was approved by the ethics committee of the First Affiliated Hospital of Xinxiang Medical University (Weihui, China). Ovarian cancer tissues $(n=58)$ and their matched adjacent normal tissues were collected from 58 patients with ovarian cancer from the First Affiliated Hospital of Xinxiang Medical University between September 2014 and April 2016. The patients were between 44 and 68 years old, with a mean age of 57.7 years. Written informed consent was obtained from all patients. No patients received radiation therapy or chemotherapy prior to surgical resection. The tissues were immediately snap-frozen in liquid nitrogen following surgical removal and stored until use. The clinical characteristics of patients, as determined using tumor, node, metastasis staging are summarized in Table I (22). Patients were included in the present study if they exhibited primary ovarian cancer and were excluded if they had received radiation therapy or chemotherapy prior to surgical resection. In addition, all patients involved in the present study were categorized into a high miR-142-3p expression group and a low miR-142-3p expression group, based on the mean expression value (1.16) of miR-142-3p.

Cell culture. Normal human ovarian epithelial cell line IOSE386, human ovarian cancer cell lines (SKOV3, HOC1, HO-8910) and the cisplatin-resistant ovarian cancer cell line SKOV3/DDP cells were purchased from American Type Culture Collection (Manassas, VA, USA). Ovarian cancer OVAC cells were purchased from Cell Bank of Central South University (Changsha, China). The cell lines were cultured in RPMI-1640 (Hyclone; GE Healthcare Life Sciences, Logan, UT, USA) supplemented with $15 \%$ fetal bovine serum (Gibco; Thermo Fisher Scientific, Inc., Waltham, MA, USA) at $37^{\circ} \mathrm{C}$ in $5 \% \mathrm{CO}_{2}$.

Cell transfection. To study the function of miR-142-3p and SIRT1, SKOV3 and SKOV3/DDP cells were transfected with miR-142-3p mimic (cat. no. B01001), scrambled miR control (cat. no. B01001; miR-NC), negative control (NC) inhibitor (cat. no. B03001; anti-NC), miR-142-3p inhibitor (cat. no. B03001; anti-miR-142-3p; all obtained from Shanghai GenePharma Co., Ltd., Shanghai, China), or co-transfected with miR-142-3p mimic and pcDNA3.1-SIRT1 plasmid (Yearthbio, Changsha, China), or co-transfected with miR-142-3p mimic and blank pcDNA3.1 vector using Lipofectamine ${ }^{\circledR} 2000$ (Invitrogen; Thermo Fisher Scientific, Inc.), according to the manufacturer's protocol.

Reverse transcription-quantitative polymerase chain reaction (RT-qPCR). Total RNA from tissues and cell lines was extracted using RNAiso plus (Takara Bio, Inc., Otsu, Japan). RNA was then converted into cDNA on an ABI 7300 plus system (Thermo Fisher Scientific, Inc.) using a PrimeScript ${ }^{\circledR}$ RT reagent kit (Takara Bio, Inc.), according to the manufacturer's protocol.
RT-qPCR was conducted using a Mir-XTM miRNA qPCR $\mathrm{SYBR}^{\circledR}$ kit (Takara Bio, Inc.) and SYBR Premix Ex Taq II (Takara Bio, Inc.) on an ABI 7300 plus system. U6 was used as the internal reference for miR-142-3p expression. GAPDH was used as internal reference for mRNA expression. The SIRT1 primer sequences were: 5'-TAGCCTTGTCAGATAAGG AAGGA-3' and 5'-ACAGCTTCACAGTCA ACTTTGT-3'. The GAPDH primer sequences were: 5'-CTGGGCTACACT GAGCACC-3' and 5'-AAGTGGTCGTTGAGGGCAATG-3'. The primers for miR-142-3p (cat. no. HmiRQP0186) and U6 (cat. no. HmiRQP9001) were purchased from Guangzhou Fulengen Co., Ltd. (Guangzhou, China); sequences were not supplied. The PCR reaction conditions were: $95^{\circ} \mathrm{C}$ for $3 \mathrm{~min}$, followed by 40 cycles of denaturation at $95^{\circ} \mathrm{C}$ for $15 \mathrm{sec}$ and annealing/elongation at $60^{\circ} \mathrm{C}$ for $30 \mathrm{sec}$. The relative expression was analyzed using the $2^{-\Delta \Delta \mathrm{Cq}}$ method (23).

Drug sensitivity assay. The drug sensitivity of SKOV3/DDP cells was measured using a Cell Counting kit-8 (CCK)-8 assay. SKOV3/DDP cells $\left(1 \times 10^{5}\right.$ cells $\left./ \mathrm{ml}\right)$ were seeded in 96 -well plates and cultured at $37^{\circ} \mathrm{C}$ for $24 \mathrm{~h}$. Cells were treated with cisplatin (Sigma-Aldrich; Merck KGaA, Darmstadt, Germany) at various concentrations $(2,4,8,16,32,64,128,256$ and $512 \mu \mathrm{g} / \mathrm{ml})$. Following incubation at $37^{\circ} \mathrm{C}$ for $48 \mathrm{~h}, 10 \mu \mathrm{l}$ CCK-8 reagent (Sigma-Aldrich; Merck $\mathrm{KGaA}$ ) was added into each well and then cells were cultured at $37^{\circ} \mathrm{C}$ for $2 \mathrm{~h}$. The absorbance of each sample was measured at $450 \mathrm{~nm}$ using a plate reader (TECAN Infinite M200; Tecan Group, Ltd., Männedorf, Switzerland).

Western blot analysis. Tissues and all cells lines used in this study were lysed in cold radioimmunoprecipitation assay buffer (Beyotime Institute of Biotechnology, Haimen, China). Protein concentration was determined using a Bicinchoninic Acid Protein Assay kit (Pierce; Thermo Fisher Scientific, Inc.), according to the manufacturer's protocols. The protein (50 $\mu \mathrm{g}$ per lane) was separated via 12\% SDS-PAGE (Pierce; Thermo Fisher Scientific, Inc.) and then transferred onto a polyvinylidene difluoride (PVDF) membrane (Thermo Fisher Scientific, Inc.). Following blocking in 5\% non-fat dried milk in PBS at room temperature for $3 \mathrm{~h}$, the PVDF membrane was incubated with rabbit anti-SIRT1 primary antibody (1:100; ab32441; Abcam, Cambridge, MA, USA) or rabbit anti-GAPDH primary antibody (1:100; cat. no. ab9485; Abcam) at room temperature for $3 \mathrm{~h}$. Following washing with PBS with Tween-20 for $10 \mathrm{~min}$, the membrane was incubated with the horseradish peroxidase conjugated goat anti-rabbit secondary antibody (1:5,000; cat. no. ab205718; Abcam) at room temperature for $1 \mathrm{~h}$. Results were visualized using an enhanced chemiluminescence kit (Pierce; Thermo Fisher Scientific, Inc.). Protein expression levels were analyzed with Image-Pro Plus software 6.0 (Media Cybernetics, Inc., Rockville, MD, USA). GAPDH was used as the internal reference.

Cell proliferation analysis. SKOV3 cells $\left(2 \times 10^{4}\right)$ were seeded in 96-well plates, each well with $100 \mu \mathrm{l}$ of fresh serum-free medium with $0.5 \mathrm{~g} / 1$ MTT (Sigma-Aldrich; Merck KGaA). This cell line was selected as it demonstrated that the lowest expression of miR-142-3p. Following incubation at $37^{\circ} \mathrm{C}$ 
Table I. Association between miR-142-3p expression and clinicopathological characteristics of patients with ovarian cancer.

\begin{tabular}{|c|c|c|c|c|}
\hline Variable & Cases $(n=58)$ & Low miR-142-3p (n=32) & High miR-142-3p $(n=26)$ & $\mathrm{P}$-value \\
\hline Age, years & & & & 0.594 \\
\hline$\leq 55$ & 22 & 11 & 11 & \\
\hline$>55$ & 36 & 21 & 15 & \\
\hline Differentiation & & & & $0.027^{\mathrm{a}}$ \\
\hline Well/moderately & 37 & 16 & 21 & \\
\hline Poor & 21 & 16 & 5 & \\
\hline Lymph node metastasis & & & & 0.156 \\
\hline Present & 17 & 12 & 5 & \\
\hline Absent & 41 & 20 & 21 & \\
\hline Clinical stage & & & & 0.113 \\
\hline I-II & 33 & 15 & 18 & \\
\hline III-IV & 25 & 17 & 8 & \\
\hline
\end{tabular}

${ }^{\mathrm{a}} \mathrm{P}<0.05 . \mathrm{miR}$, microRNA.

for $0,24,48$ and $72 \mathrm{~h}$, the medium was removed and $50 \mu \mathrm{l}$ dimethyl sulfoxide (Sigma-Aldrich; Merck KGaA) was added. Following incubation at $37^{\circ} \mathrm{C}$ for $10 \mathrm{~min}$, the absorbance of each sample was measured at $570 \mathrm{~nm}$ using a plate reader (TECAN Infinite M200; Tecan Group, Ltd.).

Bioinformatics analysis. TargetScan version 7.1 (www. targetscan.org) was used to predict the target genes of miR-142-3p, according to the manufacturer's protocol.

Dual luciferase reporter assay. The wild type (WT) or mutant type (MT) of SIRT1 3'UTR was inserted into the multiple cloning site of the psiCHECK ${ }^{\text {TM } 2}$ vector (Promega Corporation, Madison, WI, USA). SKOV3 cells were co-transfected with $100 \mathrm{ng}$ WT-SIRT1-3'UTR or MT-SIRT1-3'UTR plasmid, and $100 \mathrm{nM}$ miR-142-3p mimic or miR-NC using Lipofectamine ${ }^{\circledR} 2000$, according to the manufacturer's protocols. Following transfection for $48 \mathrm{~h}$, the Renilla luciferase activity and firefly luciferase activity were determined using a Dual-Luciferase Reporter Assay system (Promega Corporation), according to the manufacturer's protocol. Firefly luciferase activity was normalized to Renilla luciferase activity.

Statistical analysis. All data in the present study are expressed as the mean \pm standard deviation. Statistical analysis was conducted using SPSS 19.0 (IBM Corp., Armonk, NY, USA). The difference between two groups was analyzed using Student's t-test and differences among $>2$ groups were analyzed using one-way analysis of variance, followed by a post hoc Turkey's post hot test. The association between miR-142-3p expression and clinicopathological characteristics of patients with ovarian cancer was analyzed using the Chi-square test. Pearson correlation analysis was conducted for the correlation between miR-142-3p and SIRT1 mRNA expression in ovarian cancer tissues. $\mathrm{P}<0.05$ was considered to indicate a statistically significant difference. All analyses were performed in triplicate.

\section{Results}

Downregulation of miR-142-3p in ovarian cancer is associated with poor differentiation. Firstly, RT-qPCR data revealed that miR-142-3p expression levels were significantly reduced in ovarian cancer tissues compared with in adjacent tissues (Fig. 1A). To confirm these findings, the expression of miR-142-3p in several common ovarian cancer cell lines was investigated. As demonstrated in Fig. 1B, the expression levels of miR-142-3p were significantly lower in ovarian cancer cell lines compared with in the normal human ovarian epithelial cell line IOSE386 (Fig. 1B). Thus, miR-142-3p is downregulated in ovarian cancer. In addition, patients were categorized into a high miR-142-3p expression group and low miR-142-3p expression group, based on the mean expression value of miR-142-3p. Further investigation revealed that decreased expression levels of miR-142-3p were significantly associated with poor differentiation (Table I).

miR-142-3p suppresses the proliferation and chemoresistance of ovarian cancer cells. The effect of miR-142-3p on the proliferation of ovarian cancer cells was investigated. SKOV3 cells were transfected with miR-142-3p mimic to upregulate its expression. Transfection with miR-142-3p mimic revealed a significant increase in miR-142-3p expression levels in SKOV3 cells compared with in the control group. However, transfection with miR-NC did not affect the expression of miR-142-3p in SKOV3 cells (Fig. 2A). As the transfection with miR-NC did not affect miR-142-3p expression when compared with the control group, the proliferation of SKOV3 cells in the control group was not assessed. An MTT assay was then used to analyze cell proliferation, which demonstrated that the proliferation of SKOV3 cells was significantly reduced within the miR-142-3p-transfected group at $72 \mathrm{~h}$ compared with in cells transfected with miR-NC (Fig. 2B). Thus, miR-142-3p may serve a suppressive role in ovarian cancer cell proliferation.

The role of miR-142-3p in chemoresistance of ovarian cancer cells was investigated. Cisplatin-resistant SKOV3/DDP 

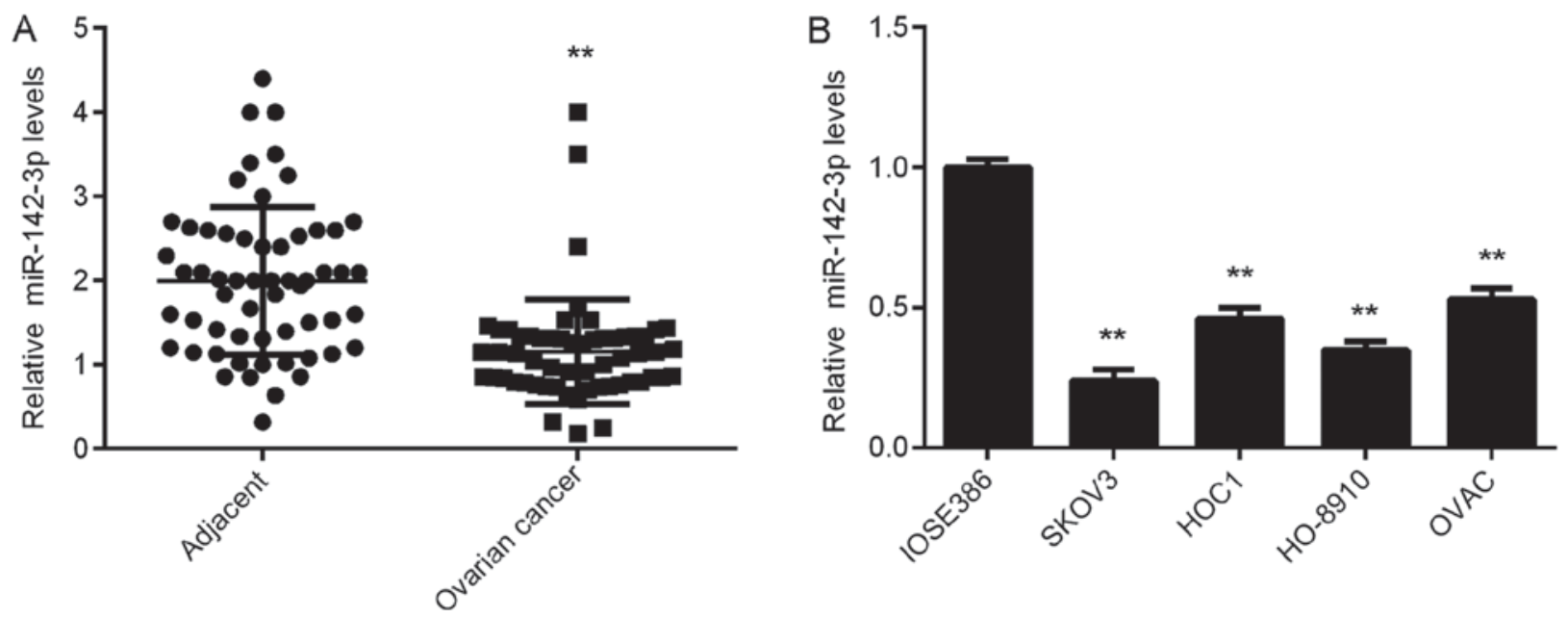

Figure 1. miR-142-3p expression in ovarian cancer. (A) miR-142-3p expression levels were reduced in ovarian cancer tissues compared with in adjacent nontumor tissues. ${ }^{* * *} \mathrm{P}<0.01$ vs. Adjacent tissues. (B) miR-142-3p expression levels were reduced in ovarian cancer cell lines compared with in the normal human ovarian epithelial cell line IOSE386. ${ }^{* *} \mathrm{P}<0.01$ vs. IOSE386 cells. miR, microRNA
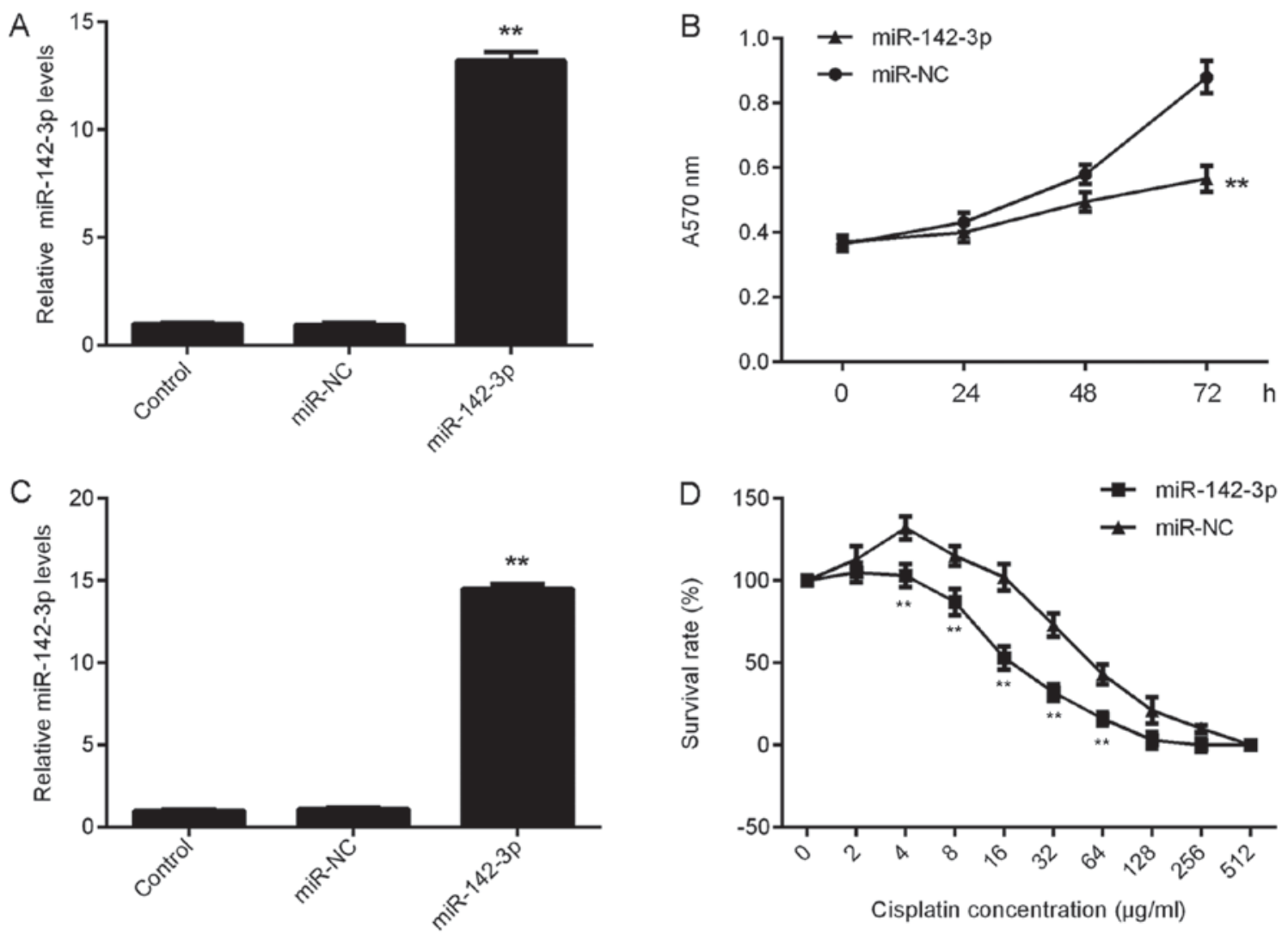

Figure 2. Effect of miR-142-3p on ovarian cancer cell proliferation and chemoresistance. SKOV3 cells were transfected with miR-142-3p mimic or miR-NC, respectively, and non-transfected SKOV3 cells were used as the control group. Post-transfection, (A) RT-qPCR was used to determine miR-142-3p expression levels. ${ }^{* *} \mathrm{P}<0.01$ vs. Control. (B) An MTT assay was conducted to examine cell proliferation. ${ }^{* *} \mathrm{P}<0.01$ vs. miR-NC. Subsequently, SKOV3/DDP cells were transfected with miR-142-3p mimic or miR-NC, respectively, and non-transfected SKOV3/DDP cells were used as the control group. Post-transfection, (C) RT-qPCR was used to determine the miR-142-3p levels. ${ }^{* *} \mathrm{P}<0.01$ vs. Control. (D) An MTT assay was conducted to examine cell proliferation. ${ }^{* *} \mathrm{P}<0.01$ vs. miR-NC. miR, microRNA; NC, negative control (scrambled); RT-qPCR, reverse transcription-quantitative polymerase chain reaction; SKOV3/DDP, cisplatin-resistant SKOV3 cells.

cells were transfected with miR-142-3p mimic or miR-NC, respectively. Following transfection, the miR-142-3p levels were significantly increased in the miR-142-3p group compared with in the control group; transfection with miR-NC demonstrated no effect on the expression of miR-142-3p in SKOV3/DDP cells (Fig. 2C). As the transfection with miR-NC did not affect miR-142-3p expression when compared with the control group, the survival rate of SKOV3/DDP cells in the control group was not assessed. A CCK-8 assay was conducted to assess the survival rate of cells treated with various concentrations of 
A

\begin{tabular}{|c|c|c|c|c|c|c|c|}
\hline & $\begin{array}{l}\text { Predicted consequential pairing of target region (top) } \\
\text { and miRNA (bottom) }\end{array}$ & $\begin{array}{l}\text { Site } \\
\text { type }\end{array}$ & $\begin{array}{c}\text { Context+- } \\
\text { score }\end{array}$ & $\begin{array}{l}\text { Context++ score } \\
\text { percentile }\end{array}$ & $\begin{array}{l}\text { Weighted } \\
\text { context+ score }\end{array}$ & $\begin{array}{c}\begin{array}{c}\text { Conserved branch } \\
\text { length }\end{array} \\
\end{array}$ & $\mathrm{P}_{\mathrm{CT}}$ \\
\hline $\begin{array}{l}\text { Position } 189-196 \text { of SIRT1 } 3 \text { UTR } \\
\text { hsa-miR-142-3p.2 }\end{array}$ & $\begin{array}{l}5^{\prime} \quad \text { W. UACUUGUACAAACUCARCACUAR } \\
\text { 3, } \\
\text { 3GGUAUUUCAUCCUUUGUGAUG }\end{array}$ & $8 \mathrm{mer}$ & -0.31 & 97 & -0.31 & 3.779 & 0.55 \\
\hline
\end{tabular}

B

WT SIRT1 3' UTR 5'...ACUUGUACAAACUCAACACUAA...3'

miR-142-3p 3'...AGGUAUUUCAUCCUUUGUGAUG...5'

MT SIRT1 3' UTR 5'...ACUUGUACAAACUCUUGUGAUA...3'

C

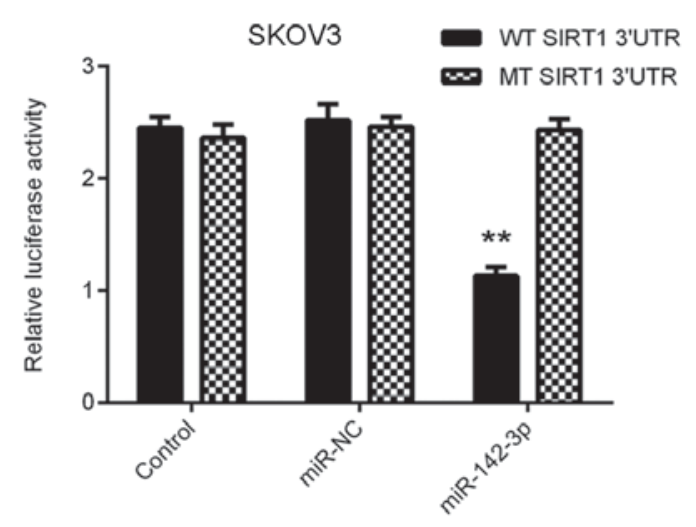

D

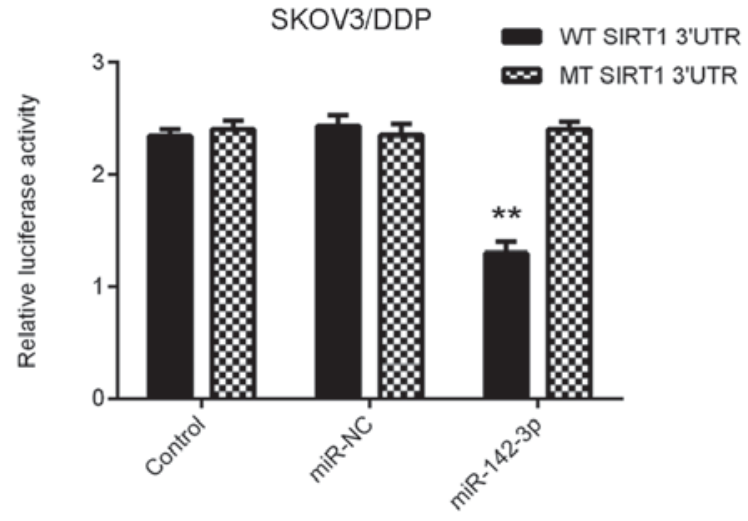

Figure 3. Identification of target gene of miR-142-3p. (A) TargetScan software indicated that SIRT1 is a target gene of miR-142-3p. (B) Luciferase reporter vectors containing WT or MT of SIRT1 3'UTR were generated. (C and D) Luciferase activity was significantly reduced in SKOV3 and SKOV3/DDP cells co-transfected with the WT-SIRT1-3'UTR plasmid and miR-142-3p mimic, but were unaltered in SKOV3 and SKOV3/DDP cells co-transfected with the MT-SIRT1-3'UTR plasmid and miR-142-3p mimic, when compared with the control group. ${ }^{* *} \mathrm{P}<0.01 \mathrm{vs.} \mathrm{Control.} \mathrm{miR,} \mathrm{microRNA;} \mathrm{SIRT1,} \mathrm{sirtuin} \mathrm{1;} \mathrm{MT,}$ mutant type; SKOV3/DDP, cisplatin-resistant SKOV3 cells; 3'UTR, 3'-untranslated region; WT, wild-type.

cisplatin. Under the same concentration of cisplatin (from 4 , $8,16,32$ and $64 \mu \mathrm{g} / \mathrm{ml}$ ), the survival rate of SKOV3/DDP cells was significantly reduced in the miR-142-3p group compared with in the miR-NC group (Fig. 2D). These findings suggested that miR-142-3p may promote the sensitivity of SKOV3/DDP cells to cisplatin.

SIRT1 is a direct target gene of miR-142-3p in ovarian cancer cells. Based on the bioinformatics analysis data, SIRT1 was reported to be a putative target gene of miR-142-3p (Fig. 3A). To confirm this prediction, luciferase vectors containing WT or MT SIRT1 3'-UTR were employed (Fig. 3B). Luciferase reporter assay data indicated that luciferase activity was significantly reduced in SKOV3 and SKOV3/DDP cells co-transfected with the WT-SIRT1-3'UTR plasmid and miR-142-3p mimic, but unaltered in SKOV3 cells co-transfected with the MT-SIRT1-3'UTR plasmid and miR-142-3p mimic, when compared with the control group (Fig. 3C and D). Therefore, miR-142-3p may bind to the 3'UTR of SIRT1 mRNA in ovarian cancer SKOV3 and SKOV3/DDP cells.

In the present study, the effect of miR-142-3p on the expression of SIRT1 in ovarian cancer cells was analyzed. As presented in Fig. 4A and B, the mRNA and protein expression levels of SIRT1 in SKOV3 and SKOV3/DDP cells were significantly reduced in the miR-142-3p group compared with in the miR-NC group. To further confirm these data, SKOV3 and SKOV3/DDP cells were transfected with miR-142-3p inhibitor or NC inhibitor. Following transfection, miR-142-3p expression levels were significantly decreased in the miR-142-3p inhibitor group compared with in the control group; however, transfection with NC inhibitor did not markedly affect miR-142-3p expression in SKOV3 and SKOV3/DDP cells (Fig. 4C). Further investigation demonstrated that the mRNA and protein expression levels of SIRT1 were significantly higher in the anti-miR-142-3p group compared with in the anti-NC group (Fig. 4D and E). Collectively, these findings indicated that miR-142-3p may inhibit SIRT1 expression by directly binding to the 3'UTR of SIRT1 mRNA in ovarian cancer cells.

SIRT1 rescues the miR-142-3p-mediated suppression of proliferation and chemoresistance of ovarian cancer cells. Based on the aforementioned findings, it was suggested that SIRT1 may be involved in miR-142-3p-mediated suppression of ovarian cancer cell proliferation and chemoresistance. To clarify this speculation, miR-142-3p-overexpressing SKOV3 cells were transfected with an SIRT1-expression plasmid to upregulate its expression, or a blank vector as the control. Following transfection, the mRNA and protein levels of SIRT1 were significantly increased in the miR-142-3p + SIRT1 group compared with in the miR-142-3p + blank group (Fig. 5A and B). MTT assay data further revealed that the proliferation of SKOV3 cells were significantly increased in the miR-142-3p + SIRT1 group compared with in the miR-142-3p + blank group at 48 and $72 \mathrm{~h}$ (Fig. 5C), indicating that SIRT1 may have rescued the suppressive effect of miR-142-3p on SKOV3 cell proliferation. Subsequently, miR-142-3p-overexpressing SKOV3/DDP 
A
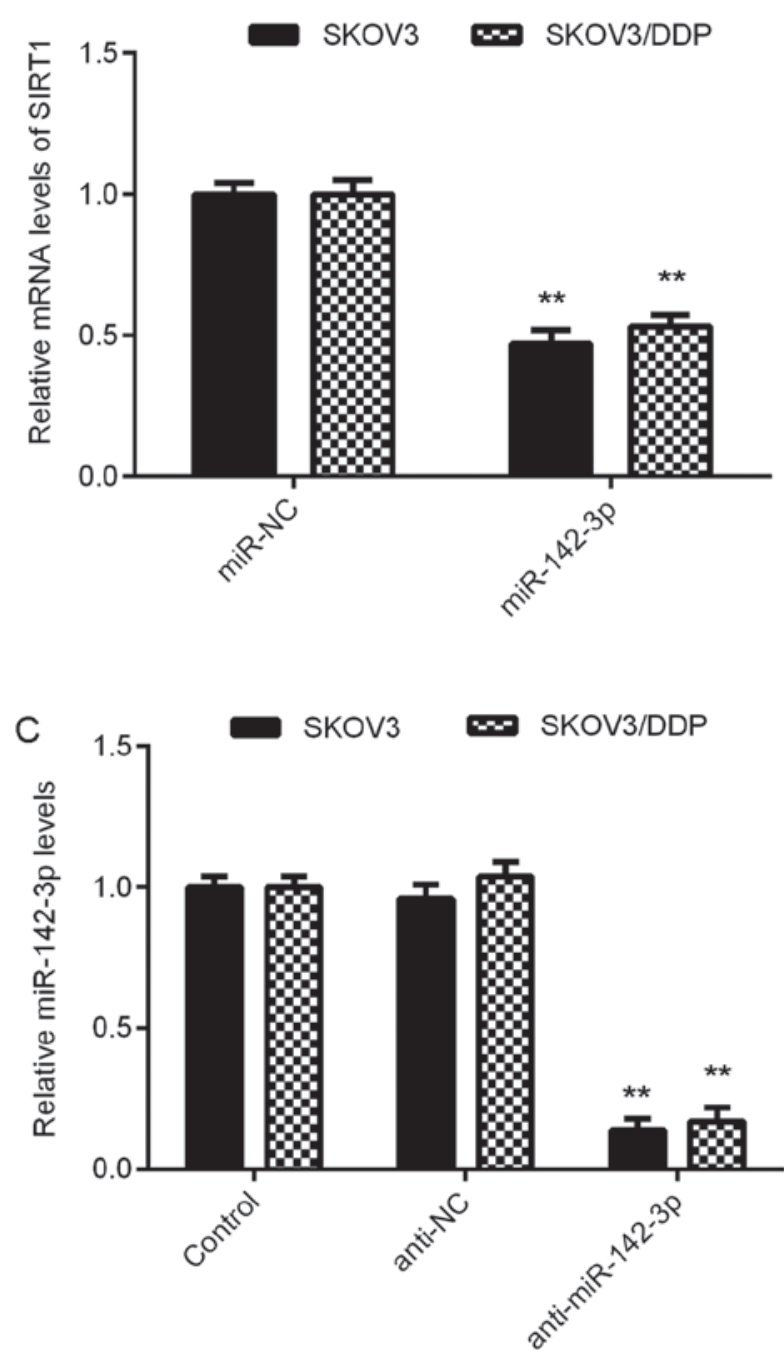

$E$

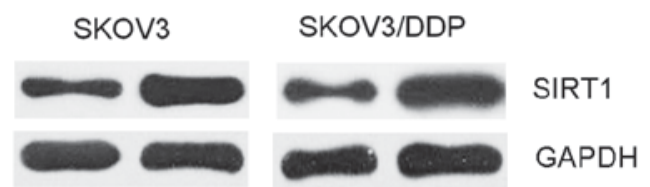

B
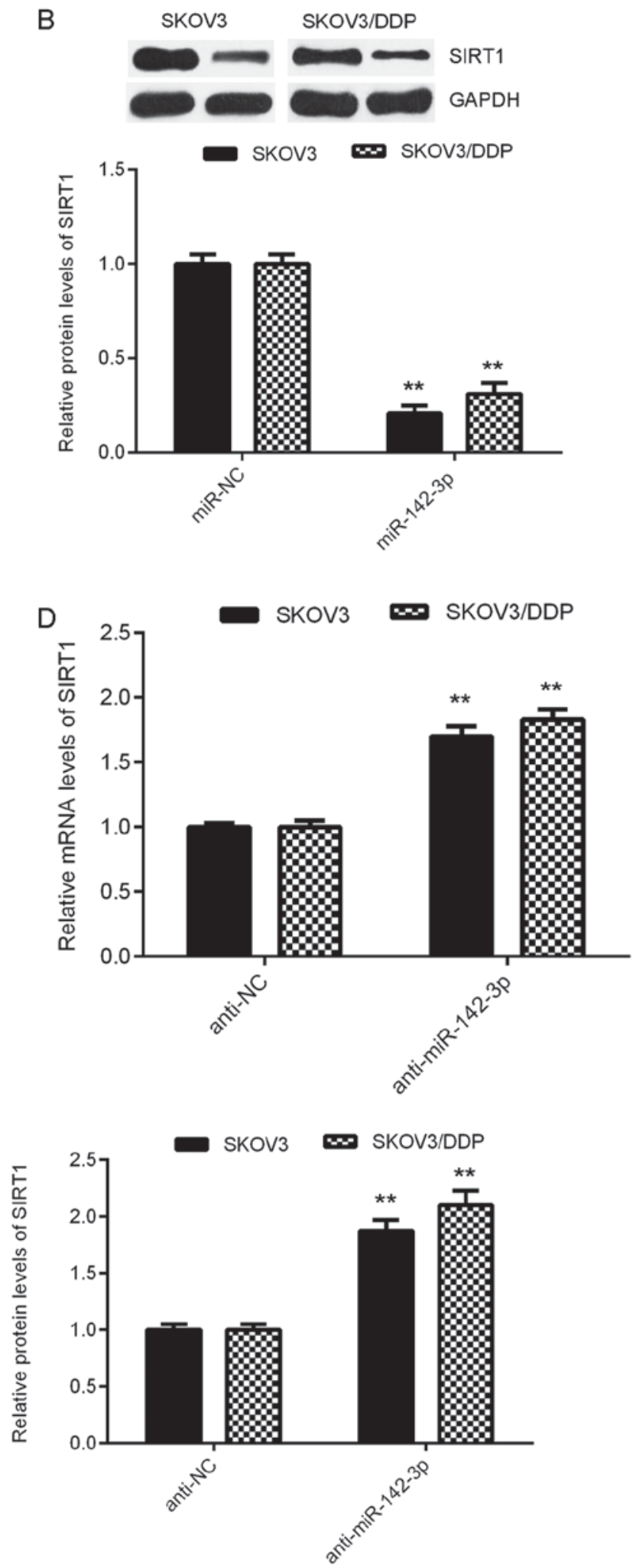

Figure 4. Effects of miR-142-3p on SIRT1 expression. (A) RT-qPCR and (B) western blotting were used to detect the mRNA and protein expression levels of SIRT1 in SKOV3 and SKOV3/DDP cells transfected with miR-142-3p mimic or miR-NC, respectively. ${ }^{* *} \mathrm{P}<0.01$ vs. miR-NC. Subsequently, SKOV3 and SKOV3/DDP cells were transfected with anti-miR-142-3p or anti-NC, respectively. Non-transfected cells were used as the control group. (C) Post-transfection, RT-qPCR was used to examine the miR-142-3p expression. ${ }^{* *} \mathrm{P}<0.01$ vs. Control. (D) RT-qPCR and (E) western blotting were used to detect the mRNA and protein expression levels of SIRT1. " $\mathrm{P}<0.01$ vs. anti-NC. miR, microRNA; miR-NC, negative control (scramble) miR; anti-miR-142-3p, miR-142-3p inhibitor; anti-NC, negative control inhibitor; RT-qPCR, reverse transcription-quantitative polymerase chain reaction; SIRT1, sirtuin 1.

cells were also transfected with an SIRT1-expression plasmid, and the mRNA and protein levels of SIRT1 were significantly upregulated following transfection (Fig. 5D and E).
A CCK-8 assay was then conducted to assess the survival rates of SKOV3/DDP cells treated with cisplatin at various concentrations. Under the same concentrations of cisplatin, 
A

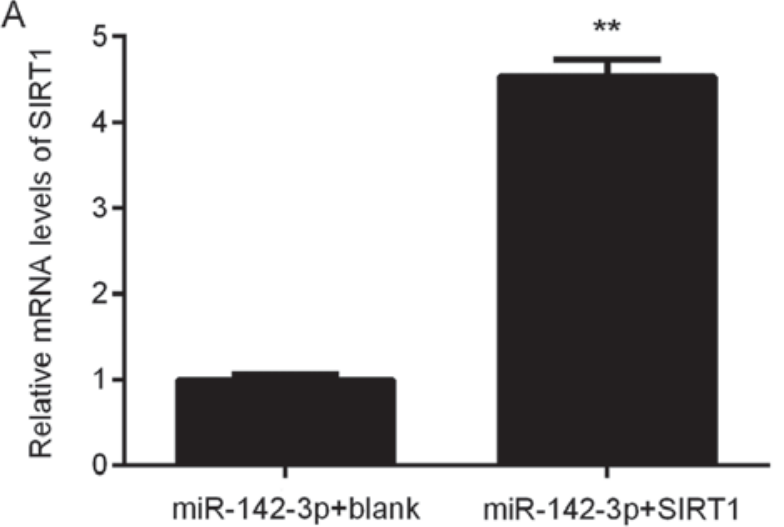

C

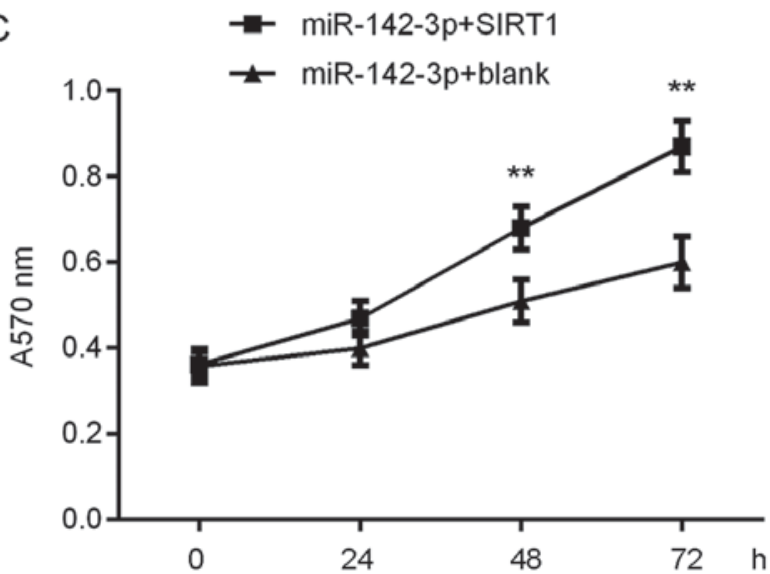

$E$

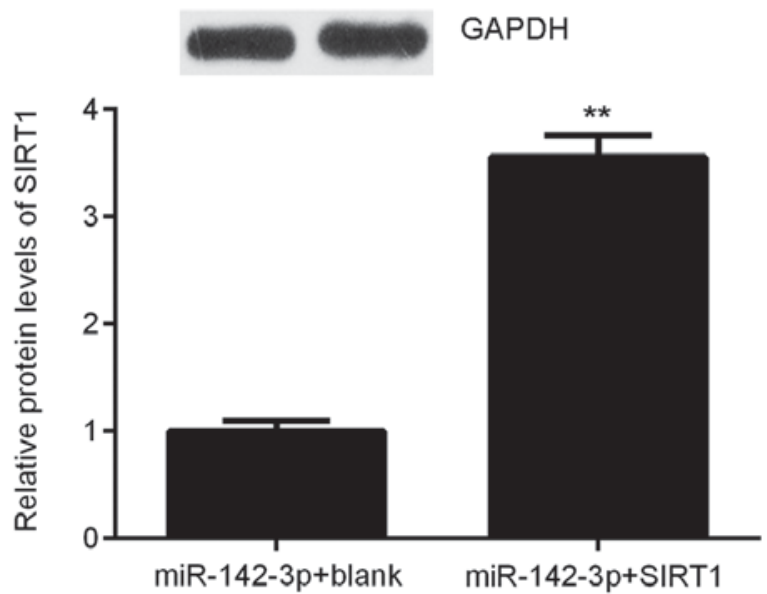

B

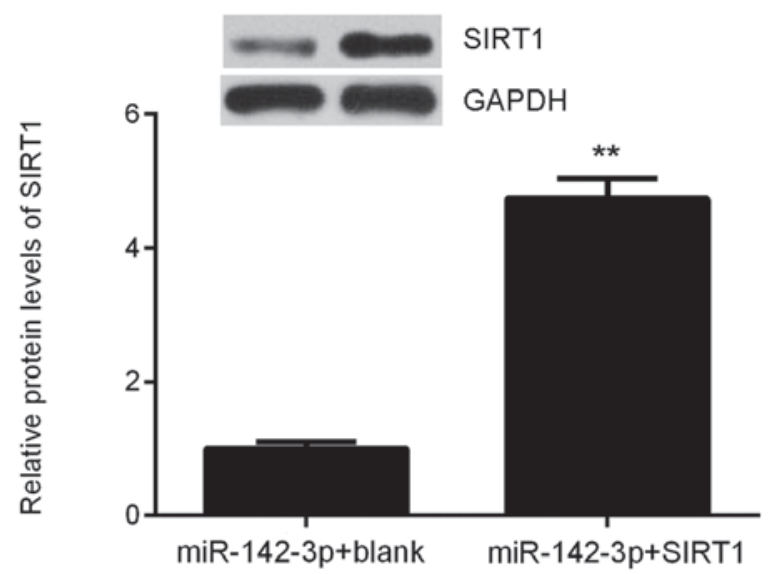

D

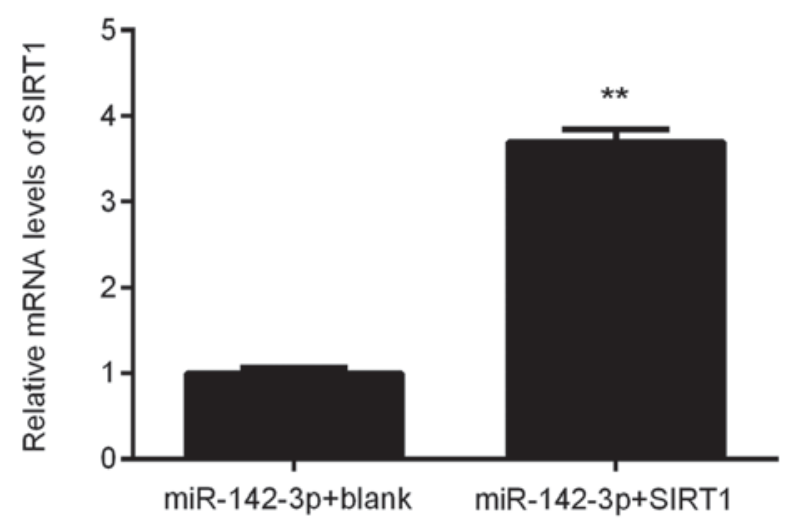

$\mathrm{F}$
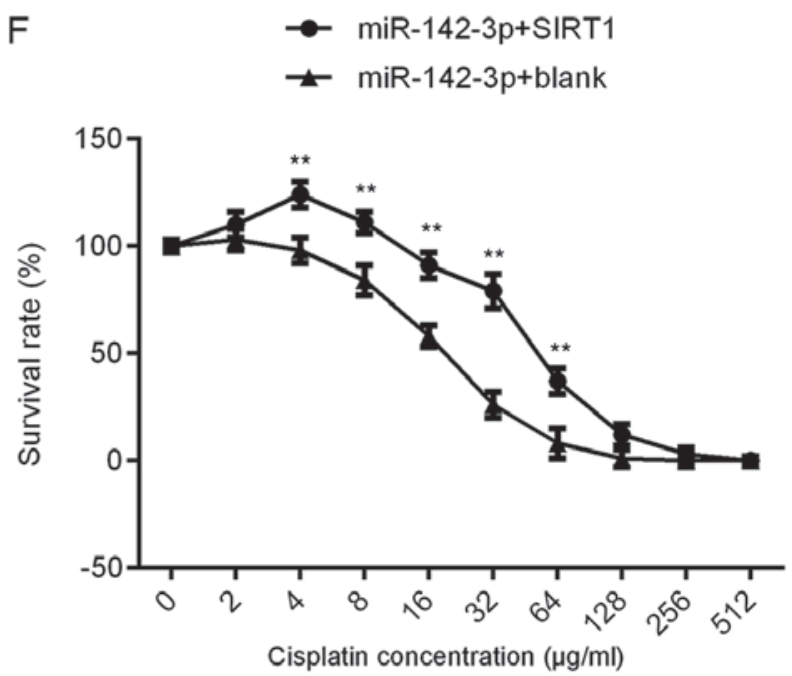

Figure 5. Effect of SIRT1 upregulation on miR-142-3p-mediated suppression of proliferation and chemoresistance of ovarian cancer cells. miR-142-3p-overexpressing SKOV3 cells were transfected with SIRT1 expression plasmid or a blank vector. Post-transfection, (A) RT-qPCR and (B) western blotting were used to detect the mRNA and protein expression of SIRT1. (C) An MTT assay was used to examine cell proliferation. Subsequently, miR-142-3p-overexpressing SKOV3/DDP cells were transfected with a SIRT1 expression plasmid or a blank vector. Post-transfection, (D) RT-qPCR and (E) western blotting was used to detect the mRNA and protein expression levels of SIRT1, and (F) a Cell Counting kit- 8 assay was used to examine drug sensitivity. ${ }^{* *} \mathrm{P}<0.01$ vs. miR1423p + blank. miR, microRNA; blank, blank vector; SIRT1, sirtuin 1; RT-qPCR, reverse transcription-quantitative polymerase chain reaction.

the survival rate was significantly higher at $4,8,16,32$ and $64 \mu \mathrm{g} / \mathrm{ml}$ in the miR-142-3p + SIRT1 group compared with in the miR-142-3p + blank group (Fig. 5F). Thus, SIRT1 rescued the miR-142-3p-mediated suppression of chemoresistance of SKOV3/DDP cells.
SIRT1 is highly upregulated in ovarian cancer. The expression of SIRT1 in ovarian cancer tissues and cell lines was detected. The results indicated that SIRT1 mRNA expression levels were significantly higher in ovarian cancer tissues compared with in adjacent tissues (Fig. 6A). Additionally, the mRNA and 

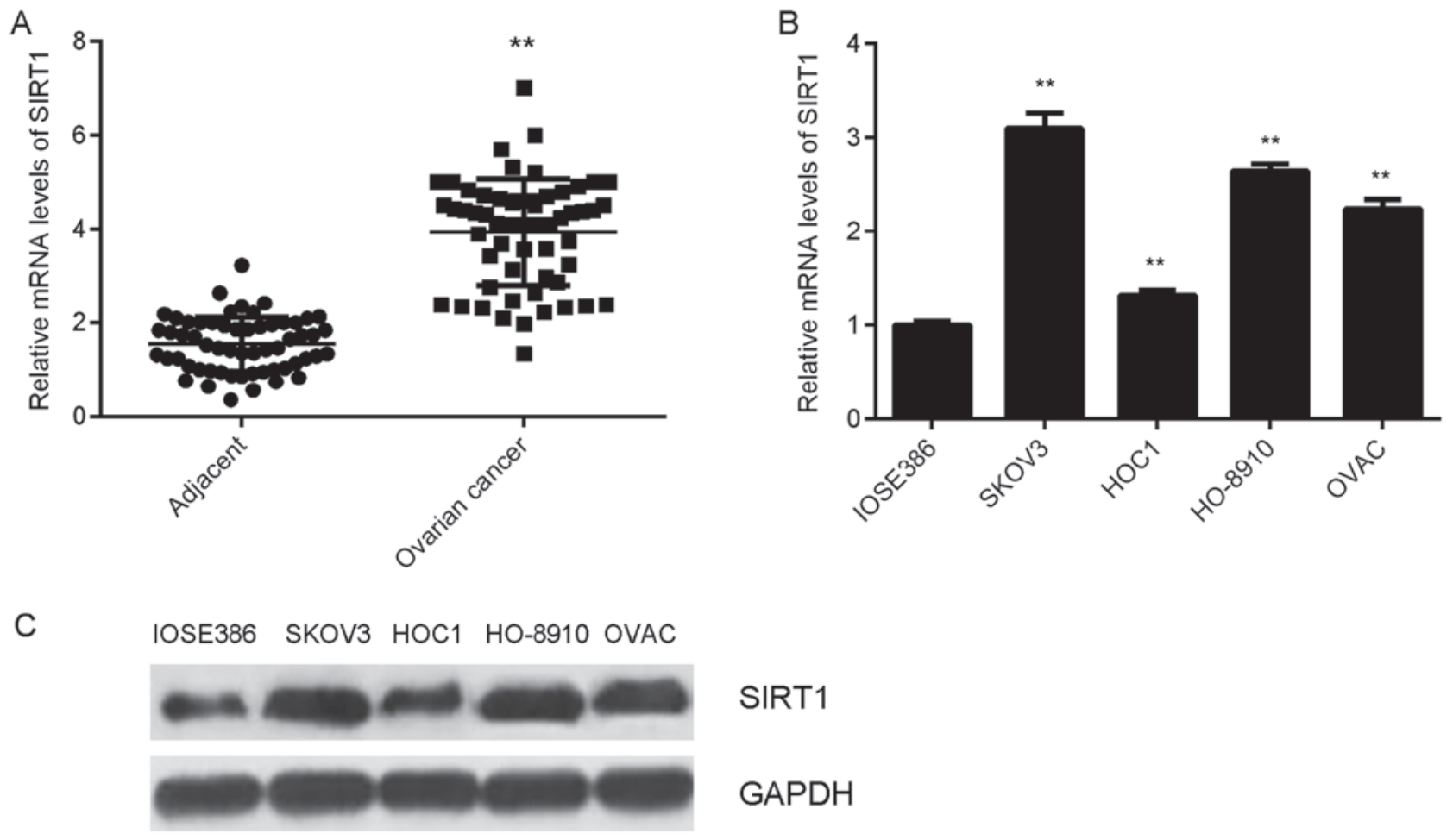

\section{SIRT1}

\section{GAPDH}

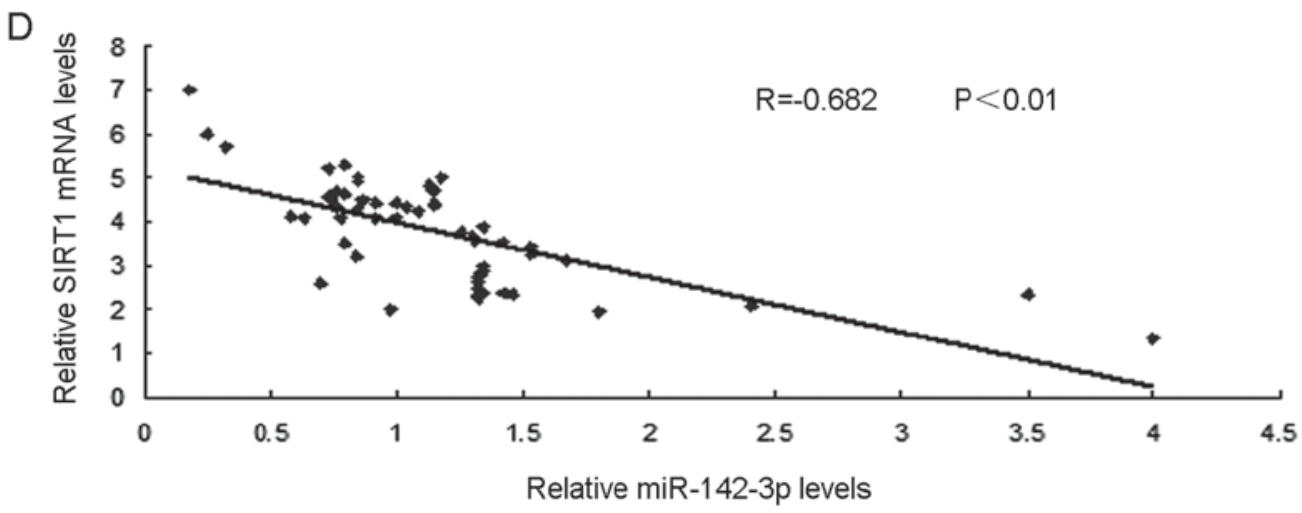

Figure 6. SIRT1 expression in ovarian cancer. (A) RT-qPCR was used to analyze the mRNA expression levels of SIRT1 in ovarian cancer tissues compared with in matched adjacent non-tumor tissues. ${ }^{* * *} \mathrm{P}<0.01$ vs. Adjacent tissues. (B) RT-qPCR and (C) western blotting were employed to examine the mRNA and protein expression levels of SIRT1 in ovarian cancer cell lines compared with in the normal human ovarian epithelial cell line IOSE386. ${ }^{* *} \mathrm{P}<0.01 \mathrm{vs.} \mathrm{IOSE386}$ cells. (D) A negative correlation between SIRT1 mRNA and miR-142-3p expression levels in ovarian cancer tissues was observed. miR, microRNA; RT-qPCR, reverse transcription-quantitative polymerase chain reaction; SIRT1, sirtuin 1.

protein expression levels of SIRT1 were significantly upregulated in ovarian cancer cell lines compared with in normal ovarian epithelial cells (Fig. 6B and C). In addition, a negative correlation between SIRT1 and miR-142-3p expression in ovarian cancer tissues was observed (Fig. 6D). These findings suggested that downregulation of miR-142-3p may contribute to the upregulation of SIRT1 in ovarian cancer.

\section{Discussion}

The regulatory mechanism of miR-142-3p underlying ovarian cancer progression and chemoresistance is largely unclear. In the present study, miR-142-3p expression levels were significantly lower in ovarian cancer tissues and cell lines, when compared with those in adjacent tissues and normal human ovarian epithelial cell line IOSE386, respectively. The reduced expression of miR-142-3p was significantly associated with advanced malignancy. Ectopic expression of miR-142-3p significantly inhibited the proliferation of ovarian cancer cells and increased the sensitivity of SKOV3/DDP cells to cisplatin. SIRT1 was identified as a target gene of miR-142-3p and its expression was negatively regulated by miR-142-3p in ovarian cancer cells. Further investigation demonstrated that SIRT1 rescued the suppressive effects of miR-142-3p on the proliferation and chemoresistance of ovarian cancer cells. In addition, SIRT1 was significantly upregulated in ovarian cancer; a negative correlation between the expression levels of SIRT1 and miR-142-3p in ovarian cancer tissues was observed.

miR-142-3p has been reported to serve a suppressive role in numerous common malignances (24). For instance, miR-142-3p inhibits the invasion of breast cancer cells by targeting Wiskott-Aldrich syndrome like, integrin aV and additional cytoskeletal elements (24). Furthermore, miR-142-3p may function as a potential tumor suppressor by directly 
targeting high mobility group box 1 in non-small-cell lung carcinoma (25). Recently, Wu et al (26) reported that the levels of serum miR-142-3p were lower in high-pathological grade of ovarian cancer than in low grade of ovarian cancer. These findings suggested that aberrant expression of miR-142-3p may be involved in the progression of ovarian cancer; miR-142-3p may serve as a serum biomarker to distinguish ovarian cancer of various grades. However, the expression levels of miR-142-3p in ovarian cancer tissues have not been previously been studied. In the present study, it was reported that miR-142-3p was significantly downregulated in ovarian cancer tissues and cell lines, and the reduced expression of miR-142-3p was associated with poor differentiation in ovarian cancer, suggesting that its downregulation may contribute to the malignant progression of ovarian cancer. Further investigation revealed that restoration of miR-142-3p expression significantly inhibited the proliferation of SKOV3 cells.

During chemotherapy, ovarian cancer cells are prone to drug resistance, which is associated with cancer recurrence and mortality (2). Thus, investigation into the molecular mechanism underlying chemoresistance in ovarian cancer is urgently required for identifying novel and effective therapeutic targets. In the present study, the overexpression of miR-142-3p significantly inhibited the resistance of cisplatin-resistant SKOV3/DDP cells. Similarly, miR-142-3p was previously reported to improve the drug sensitivity of acute myelogenous leukemia and NSCLC $(27,28)$.

Subsequently, bioinformatics analysis and a luciferase reporter assay were performed to study the potential targets of miR-142-3p in ovarian cancer cells. The data of the present study indicated that SIRT1 may be a direct target gene of miR-142-3p, and its expression was negatively mediated by miR-142-3p in SKOV3 and SKOV3/DDP cells. SIRT1 has been demonstrated to regulate various cellular functions including DNA repair, cell survival and metabolism via the deacetylation of target proteins such as histone and p53 (29). Recently, Asaka et al (29) reported that SIRT1 promoted the growth and cisplatin resistance of endometrial carcinoma cells. Previous studies have demonstrated that the expression levels of SIRT1 were increased in cisplatin-resistant ovarian cancer tissues compared with in cisplatin-sensitive ovarian cancer tissues; SIRT1 significantly enhanced the proliferation, chemoresistance and aggressiveness of ovarian cancer cells by upregulating numerous antioxidant pathways to inhibit oxidative stress $(21,30)$. Therefore, the results of the present study suggested that the suppressive effects of miR-142-3p on the proliferation and chemoresistance of ovarian cancer cells may have occurred via the inhibition of SIRT1 expression.

SIRT1 was previously reported to be significantly upregulated in malignant ovarian epithelial tumors compared with in benign and borderline epithelial tumors (30). Similarly, SIRT1 expression levels were upregulated in ovarian cancer tissues and cell lines compared with normal ovarian tissues and cells in the present study; a negative correlation between SIRT1 and miR-142-3p expression levels in ovarian cancer tissues was also observed. This suggested that upregulation of SIRT1 may be due to the decreased expression of miR-142-3p.

In conclusion, miR-142-3p, which is significantly downregulated in ovarian cancer, may serve a suppressive role in the proliferation and chemoresistance of ovarian cancer cells, at least partially via the direct targeting of SIRT1. Therefore, the findings of the present study suggest that miR-142-3p may be a promising therapeutic candidate for the treatment of ovarian cancer.

\section{Acknowledgements}

Not applicable.

\section{Funding}

No funding was received.

\section{Availability of data and materials}

All data generated or analyzed during this study are included in this published article.

\section{Authors' contributions}

JG performed statistical analysis, and wrote and submitted the manuscript. ZD designed the study and revised the manuscript. NW, XL, YX, YC and SL performed cellular and molecular experiments.

\section{Ethics approval and consent to participate}

This study was approved by the First Affiliated Hospital of Xinxiang Medical University (Weihui, China). Written informed consent was obtained from all patients.

\section{Consent for publication}

Written informed consent was obtained from all patients.

\section{Competing interests}

The authors declare that they have no competing interests.

\section{References}

1. Siegel RL, Miller KD and Jemal A: Cancer statistics, 2015. CA Cancer J Clin 65: 5-29, 2015.

2. Sun Y, Liu JH, Jin L, Sui YX, Han LL and Huang Y: Effect of autophagy-related beclin 1 on sensitivity of cisplatin-resistant ovarian cancer cells to chemotherapeutic agents. Asian Pac J Cancer Prev 16: 2785-2791, 2015.

3. Yu PN, Yan MD, Lai HC, Huang RL, Chou YC, Lin WC, Yeh LT and Lin YW: Downregulation of miR-29 contributes to cisplatin resistance of ovarian cancer cells. Int J Cancer 134: 542-551, 2014.

4. Sun Y, Liu JH, Jin L, Sui YX, Lai L and Yang Y: Inhibition of Beclin 1 expression enhances cisplatin-induced apoptosis through a mitochondrial-dependent pathway in human ovarian cancer SKOV3/DDP cells. Oncol Res 21: 261-269, 2014

5. Miao Y, Lu M, Yan Q, Li S and Feng Y: Inhibition of proliferation, migration, and invasion by knockdown of pyruvate kinase-M2 (PKM2) in ovarian cancer SKOV3 and OVCAR3 cells. Oncol Res 24: 463-475, 2016.

6. Teng Y, Zuo X, Hou M, Zhang Y, Li C, Luo W and Li X: A Double-negative feedback interaction between MicroRNA-29b and DNMT3A/3B contributes to ovarian cancer progression. Cell Physiol Biochem 39: 2341-2352, 2016.

7. Ambros V: The functions of animal microRNAs. Nature 431: 350-355, 2004.

8. Bartel DP: MicroRNAs: Genomics, biogenesis, mechanism, and function. Cell 116: 281-297, 2004. 
9. John B, Enright AJ, Aravin A, Tuschl T, Sander C and Marks DS: Human MicroRNA targets. PLoS Biol 2: e363, 2004.

10. Jiang Z, Zhang Y, Cao R, Li L, Zhong K, Chen Q and Xiao J: miR-5195-3p inhibits proliferation and invasion of human bladder cancer cells by directly targeting oncogene KLF5. Oncol Res 25: 1081-1087, 2017.

11. Chen X and Chen J: MiR-3188 regulates cell proliferation, apoptosis, and migration in breast cancer by targeting TUSC5 and regulating the p38 MAPK signaling pathway. Oncol Res: May 26, 2017 (Epub ahead of print).

12. Zhou Y, Yang C, Wang K, Liu X and Liu Q: MicroRNA-33b inhibits the proliferation and migration of osteosarcoma cells via targeting hypoxia-inducible factor-1 $\alpha$. Oncol Res 25: 397-405, 2017.

13. Yang M,Zhai X, Ge T, Yang C and Lou G: MiR-181a-5p Promotes proliferation and invasion, and inhibits apoptosis of cervical cancer cells via regulating inositol polyphosphate-5-phosphatase A (INPP5A). Oncol Res: Jun 23, 2017 (Epub ahead of print).

14. Wang C, Zhou B, Liu M, Liu Y and Gao R: miR-126-5p restoration promotes cell apoptosis in cervical cancer by targeting Bcl212. Oncol Res 25: 463-470, 2017.

15. Li H, Xiang Z, Liu Y, Xu B and Tang J: MicroRNA-133b inhibits proliferation, cellular migration, and invasion via targeting LASP1 in hepatocarcinoma cells. Oncol Res 25: 1269-1282, 2017.

16. Colamaio M, Puca F, Ragozzino E, Gemei M, DecaussinPetrucci M, Aiello C, Bastos AU, Federico A, Chiappetta G, Del Vecchio L, et al: miR-142-3p down-regulation contributes to thyroid follicular tumorigenesis by targeting ASH1L and MLL1. J Clin Endocrinol Metab 100: E59-E69, 2015.

17. Ghanbari R, Mosakhani N, Asadi J, Nouraee N, Mowla SJ, Yazdani Y, Mohamadkhani A, Poustchi H, Knuutila S and Malekzadeh R: Downregulation of plasma MiR-142-3p and MiR-26a-5p in patients with colorectal carcinoma. Iran J Cancer Prev 8: e2329, 2015

18. Zhang J, Shan WF, Jin TT, Wu GQ, Xiong XX, Jin HY and Zhu SM: Propofol exerts anti-hepatocellular carcinoma by microvesicle-mediated transfer of miR-142-3p from macrophage to cancer cells. J Transl Med 12: 279, 2014.

19. Ramis MR, Esteban S, Miralles A, Tan DX and Reiter RJ: Caloric restriction, resveratrol and melatonin: Role of SIRT1 and implications for aging and related-diseases. Mech Ageing Dev 146-148: 28-41, 2015.

20. Shuang T, Wang M, Zhou Y and Shi C: Over-expression of Sirt1 contributes to chemoresistance and indicates poor prognosis in serous epithelial ovarian cancer (EOC). Med Oncol 32: 260 , 2015 .
21. Mvunta DH, Miyamoto T, Asaka R, Yamada Y, Ando H, Higuchi S, Ida K, Kashima H and Shiozawa T: SIRT1 regulates the chemoresistance and invasiveness of ovarian carcinoma cells. Transl Oncol 10: 621-631, 2017.

22. Javadi S, Ganeshan DM, Qayyum A, Iyer RB and Bhosale P: Ovarian cancer, the revised FIGO staging system, and the role of imaging. AJR Am J Roentgenol 206: 1351-1360, 2016.

23. Livak KJ and Schmittgen TD: Analysis of relative gene expression data using real-time quantitative PCR and the 2(-Delta Delta C(T)) method. Methods 25: 402-408, 2001.

24. Schwickert A, Weghake E, Brüggemann K, Engbers A, Brinkmann BF, Kemper B, Seggewiß J, Stock C, Ebnet K, Kiesel L, et al: microRNA miR-142-3p inhibits breast cancer cell invasiveness by synchronous targeting of WASL, integrin alpha V, and additional cytoskeletal elements. PLoS One 10: e0143993, 2015.

25. Xiao P and Liu WL: MiR-142-3p functions as a potential tumor suppressor directly targeting HMGB1 in non-small-cell lung carcinoma. Int J Clin Exp Pathol 8: 10800-10807, 2015.

26. Wu X, Zhi X, Liu M, Xie J and Zhao S: [Elevated levels of dendritic cell-correlated miRNAs in ascites and sera of patients with ovarian cancer]. Xi Bao Yu Fen Zi Mian Yi Xue Za Zhi 31: 383-386, 2015.

27. Zhang Y, Liu Y and Xu X: Upregulation of miR-142-3p improves drug sensitivity of acute myelogenous leukemia through reducing P-glycoprotein and repressing autophagy by targeting HMGB1. Transl Oncol 10: 410-418, 2017.

28. Chen Y, Zhou X, Qiao J and Bao A: MiR-142-3p overexpression increases chemo-sensitivity of NSCLC by inhibiting HMGB1mediated autophagy. Cell Physiol Biochem 41: 1370-1382, 2017.

29. Asaka R, Miyamoto T, Yamada Y, Ando H, Mvunta DH, Kobara $\mathrm{H}$ and Shiozawa T: Sirtuin 1 promotes the growth and cisplatin resistance of endometrial carcinoma cells: A novel therapeutic target. Lab Invest 95: 1363-1373, 2015.

30. Li D, Wu QJ, Bi FF, Chen SL, Zhou YM, Zhao Y and Yang Q: Effect of the BRCA1-SIRT1-EGFR axis on cisplatin sensitivity in ovarian cancer. Am J Transl Res 8: 1601-1608, 2016.

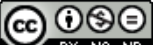

$$
\begin{aligned}
& \text { This work is licensed under a Creative Commons } \\
& \text { Attribution-NonCommercial-NoDerivatives } 4.0 \\
& \text { International (CC BY-NC-ND 4.0) License. }
\end{aligned}
$$

